\title{
OPINION
}

\section{Nanomedicine and Society}

\author{
M Ferrari $^{1}$, MA Philibert ${ }^{2}$ and WR Sanhai ${ }^{3}$
}

\section{Nanomedicine is a reality of medical research and clinical practice, and it offers new and promising approaches to fundamental problems in medicine. Most prominent are the early detection of neoplastic disease and the individualized treatment of metastases. These potentially transformational developments require careful scrutiny of the potential impact of nanomedicine on society, so that the community can guide its deployment in keeping with the fundamental tenets of medical ethics.}

Definitions of nanotechnology abound, including several used by US and international agencies. The various definitions have three substantially common elements: nanotechnological devices are at least partly man-made, have dimensions on the scale of 1 to $1,000 \mathrm{~nm}$, and possess novel "emerging" properties stemming from their nanoscale dimension-that is, properties not present in identical devices of smaller or larger dimensions. The existence of a mechanism-based, predictive proof of the causal necessity of the new properties is also required by some authors as part of the definition of nanotechnology. In the working definition we use in this article, nanomedicine is the application of nanotechnology to medicine, from basic research to disease diagnosis to therapeutic intervention, monitoring disease progression and patient management, including qualityof-life measures.

Although this article neither subscribes to a consensus definition nor provides one for full adoption, it is consistent with the ones in common use, such as the one used by the National Nanotechnology Initiative ${ }^{1}$ (to reduce ambiguity and misinterpretation). Furthermore, in the context of regulatory issues, as the science evolves and the US Food and Drug Administration (FDA) learns more about the interaction of nanoscale materials with complex biological systems, the agency may develop formal, fixed definitions, appropriately tailored to the regulation of nanoscale materials in FDA-regulated products. ${ }^{2}$

The promise of personalized nanomedicine was based on the understanding that each individual possesses a unique genetic profile predisposing him or her to respond to therapies differently. Now, armed with the predictive power of in silico models of patient populations, whole-genome testing, clinically qualified biomarkers that can assess individual responses to therapies, and other tools, the biomedical field is poised for significant advances and benefits to individuals rather than to their population mean. Nanomedicine, like no other field in science, has the potential of interpretative flexibility-flexibility in how scientists design, analyze, and interpret outcomes. The marriage of genomics, postgenomics, and nanomedicine offers the potential for disruptive innovation and therapies targeted to an individual.

"Nanomedicine" is a broad term that encompasses the development of sensors for single-molecule detection, identification of biomarkers, nanoparticles and nanocarriers for the detection and imaging of cancers, and the delivery of therapeutic molecules. Nanoparticle-based vehicles have also been used to deliver recombinant proteins as vaccines. In the area of therapeutic and imaging modalities, the overwhelming majority of recent developments have been aimed at cancer. Materials that have been investigated for their utility include soft and liquid polymers, polymers based on sugars or amino acids or both, liposomes, dendrimers, and metalloid/metallic and solid/hollow native or organically modified silicas. Such nanomaterials may be either persistent or biodegradable.

Despite the wide range of materials and potential applications, most medicinal nanoparticles are essentially targeted carriers or "smart" polymers that deliver drugs, imaging agents, and/or chemicals that disrupt growth of cancer cells. ${ }^{3}$ Some nanomaterials use peptides and other ligands to actively target the appropriate site, ${ }^{4}$ and others are passively targeted by exploiting the pathophysiological changes that occur in increased permeability of blood vessels or other cellular phenomena. The use of targeting antibodies, peptides, and other targeting moieties on the surface lends itself to personalized medicine. The fast-approaching capability to quickly identify unique surface markers on tumor cells and to design or fabricate a tailored nanomaterial that will attach

${ }^{1}$ Division of Nanomedicine, University of Texas Houston Health Science Center at Houston, Houston, Texas, USA; ${ }^{2}$ Department of Toxicology, University of Michigan School of Public Health, Ann Arbor, Michigan, USA; ${ }^{3}$ Office of the Commissioner, US Food and Drug Administration, Rockville, Maryland, USA. Correspondence: WR Sanhai (wendy.sanhai@fda.hhs.gov) 
itself with high affinity and selectivity will further enhance the attractiveness of using nanoparticles in imaging and therapeutic modalities. Moreover, several of these nanostructures lend themselves to screening in situ. Because many of the imaging nanomaterials, and all the therapeutic ones, are designed for launching to various compartments within the body, several consequent physiological challenges arise. Chief among these challenges is the somewhat predictable interplay between a complex bionanoparticle and the immune system.

All the advantages of this approach notwithstanding - and precisely because most of the useful therapeutic and nanoparticles (5-150 $\mathrm{nm}$ diameter) range in size from that of a typical rhinovirus ( $\sim 20 \mathrm{~nm}$ diameter) to that of the pox virus Molluscum contagiosum ( $200 \mathrm{~nm}$ diameter) - they are targeted by the immune system of the host. The potential for accumulation of complex, bioactive mixtures of nanomaterial and adherent peptides raises questions about acute or chronic idiopathic adverse reactions that may constitute a rare or underdiagnosed condition. Certainly, the era of nanomedicine will require extensive characterization of the therapeutic index during development and vigilant aftermarket surveillance.

On the side of the potential beneficial impact on society, considerations about the importance of nanomedicine must be placed in the context of the health problems they address. Focusing attention on oncology, it is recalled that in the United States a person dies of cancer approximately every minute, and the worldwide number is more than five times that. The dimensions of the tragedy have not decreased in recent years in the United States and Europe, despite extraordinary advances in the molecular-level understanding of the disease. Two crucial reasons for this failure to translate to basic progress in the clinic are: (i) the current inability to detect the disease at its earliest stages, when treatments are most effective and have the fewest adverse side effects, and (ii) the current inability to treat metastatic disease - in particular, due to the extraordinary, time-variable diversity of molecular connotations with which it manifests itself, even in a single person, in the course of neoplastic disease.

These are exactly the two fronts on which nanomedicine provides its most promising weaponry. ${ }^{5}$ On the side of early detection, nanomedicine is evolving a host of new approaches that will allow for the rapid, non- or minimally invasive and inexpensive testing of a very large number of molecular signatures from biological fluids. Together with the development of new, molecularly targeted nanoscale contrast agents for radiological imaging, these are envisioned to aid in the birth of a new era of medical care, in which cancer screening is available to all and affordable for all. In view of the variability and diversity of the molecular presentations of metastatic disease, not only is the notion of "personalizing" treatment a good idea; it is a probably a necessary route to the eradication of death and suffering due to cancer. The need is even more radical: beyond personalization, what is required in many cases is treatment individualization, at the level of the individual lesion. Here nanotechnology offers the opportunity to develop new approaches, individualizing treatment simultaneously on three fronts: (i) the bioactive payload, which can be, e.g., a "molecularly targeted" drug, short interfering RNA, or microRNA; (ii) the design of the carrier nanoparticle, which could be tailored to the immune responses of a patient; and, again, (iii) the design of the carrier particulate system, which can be tailored so as to optimize the likelihood of selective concentration in the target lesion. ${ }^{6}$

Research with the intent of translation into the clinic may be viewed within the classical medical ethics framework of Childress and Beauchamp. This perspective was used by the first author of this article in his presentation to the US President's Council on Bioethics on 29 June 2007 , in the first (and so far only) session dedicated by the council to matters of nanotechnology and nanomedicine. ${ }^{7}$ The presentation reflected the four cardinal dimensions of analysis in the Beauchamp-Childress system: beneficence (the utilitarian perspective of maximizing community benefit), nonmaleficence (the Hippocratic mandate of "First, do no harm"), respect (including autonomy, or the patient's right to decide), and justice (including fair access to health care).

The main conclusions of the presentation are summarized as follows. Nanotechnology offers extraordinary opportunities for medical advances and, most important, offers new hope for early detection and individualized therapy of disease. Environmental risks from nanomedicine are very modest. Nanotechnology-enabled personalized medicine poses ethical questions of autonomy and privacy that must be discussed in the broadest community context for proper policy decisions to be made.

Nanomedicine is at risk of being available only to privileged societies, at least initially, but it offers unexplored opportunities for medical advances that would benefit underprivileged populations. The final, overarching conclusion is that careful scrutiny of potential safety risks is absolutely necessary-yet the greatest risk in nanomedicine may well be in letting our concerns paralyze our action and not taking advantage of the full, revolutionary potential that nanotechnology in medicine can offer humankind. We fully echo this conclusion.

\section{ACKNOWLEDGMENT}

The views expressed herein are those of the authors and may not reflect policies of the FDA.

\section{CONFLICT OF INTEREST}

The authors declared no conflict of interest.

\section{(C) 2009 ASCPT}

1. National Nanotechnology Initiative. What Is Nanotechnology? < http://www.nano.gov/html/ facts/whatlsNano.html>.

2. US Food and Drug Administration. Nanotechnology: A Report of the U.S. Food and Drug Administration Nanotechnology Task Force <http://www.fda.gov/nanotechnology/ taskforce/report2007.html> (25 July 2007).

3. Nie, S., Xing, Y., Kim, G.J. \& Simons, J.W. Nanotechnology applications in cancer. Annu. Rev. Biomed. Eng. 9, 257-288 (2007).

4. Bharali, D.J. et al. Novel nanoparticles for the delivery of recombinant hepatitis $B$ vaccine. Nanomedicine 4, 311-317 (2008).

5. Ferrari, M. Cancer nanotechnology: opportunities and challenges. Nat. Rev. Cancer $\mathbf{5}$, 161-171 (2005).

6. Tasciotti, E. et al. Mesoporous silicon particles as a multistage delivery system for imaging and therapeutic applications. Nat. Nanotechnol. 3, 151-157 (2008).

7. The President's Council on Bioethics. Session 5: Nanotechnology, Medicine, and Ethics <http://www.bioethics.gov/transcripts/june07/ session5.html> (29 June 2007). 